\title{
Cytokeratin 18 Is Not Required for Morphogenesis of Developing Prostates but Contributes to Adult Prostate Regeneration
}

\author{
Chenlu Zhang, ${ }^{1,2}$ Yanjing Guo,, ${ }^{1,2}$ Jian Cui, ${ }^{1,2}$ Helen He Zhu, ${ }^{1,2}$ and Wei-Qiang Gao ${ }^{1,2}$ \\ ${ }^{1}$ State Key Laboratory of Oncogenes and Related Genes, Stem Cell Research Center, Renji Hospital, 160 Pu Jian Road, \\ Shanghai Jiao Tong University School of Medicine, Shanghai 200127, China \\ ${ }^{2}$ Med-X Research Institute, Shanghai Jiao Tong University, Shanghai 200030, China
}

Correspondence should be addressed to Helen He Zhu; zhuhecrane@shsmu.edu.cn and Wei-Qiang Gao; gao.weiqiang@sjtu.edu.cn Received 30 April 2013; Accepted 17 October 2013

Academic Editor: Paul Crispen

Copyright @ 2013 Chenlu Zhang et al. This is an open access article distributed under the Creative Commons Attribution License, which permits unrestricted use, distribution, and reproduction in any medium, provided the original work is properly cited.

\begin{abstract}
Cytokeratin 18 (CK18) is a key component of keratin-containing intermediate filaments and has long been used as a classic luminal cell marker in prostatic tissue. However, the in vivo function of CK18 in prostate is not known so far. We reported in this study, unexpectedly, that deletion of CK18 in a mouse model did not affect the morphological or the histological structures of adult prostate, as the CK18 knockout prostate displayed a normal glandular ductal structure, branching pattern, and composition of both luminal and basal cells. However, CK18 loss compromised the regenerative tubular branching in dorsolateral prostate after castration and androgen replacement. Therefore, in contrast to its importance as luminal cell marker, CK18 is dispensable for the prostate morphogenesis but contributes to adult prostate regeneration.
\end{abstract}

\section{Introduction}

The glandular epithelium of prostate is composed of three types of differentiated cells: luminal cells, basal cells, and rare neuroendocrine cells. Different keratins are preferentially expressed in specific prostatic epithelial cells and are routinely used as markers for respective epithelial lineages. The rectangular lumen-lining luminal cells constitute the major part of the prostate epithelial and express cytokeratin 18 (CK18), cytokeratin 8 (CK8), and androgen receptor (AR) [1], while the small and oblong basal cells frequently found adjacent to the basement membrane are positive for p63, CK5, and CK14 but do not express CK8, CK18, or AR. Deletion of basal cell maker p63 in mouse model led to absence of basal cells in prostate explants [2-4].

CK18 is a type II cytokeratin and usually coexists with type I cytokeratin CK8 to produce keratin-containing intermediate filaments [5]. Keratin intermediate filaments form cage-like structures around the nucleus, which play an essential role in the maintenance of nuclear integrity. Therefore,
CK18 acts as an important scaffold protein in response to external stresses. It is also involved in regulation of cellular processes including apoptosis, mitosis, and cell cycle [5]. Although homozygous CK18 knockout mice are viable and fertile, liver disorders with CK8-positive aggregates are found in aged $\mathrm{CK} 18^{-/-}$animals [6]. In contrast to the importance of CK18 as a luminal cell marker, its in vivo function in the prostate morphogenesis and regeneration remains unexplored so far.

The mouse prostate development initiates from the urogenital sinus (UGS) [7]. The prostatic epithelium buds out around embryonic day 17.5 (E17.5) and elongates and branches after birth. The branching morphogenesis almost completes at postnatal week two but fully matures by 60 to 90 days $[7,8]$. The adult prostate is composed of three symmetrical lobes: the anterior prostate (AP), ventral prostate (VP), and dorsolateral prostate (DLP) [9]. Prostate development, branching, and maintaining rely on serial androgen release and response. Deprivation of androgen induces intensive atrophy of prostate lobes, while androgen replacement 
efficiently stimulates prostate regeneration [10, 11]. This atrophy-regeneration process can be repeated around thirty times in rats in support of the notion that stem-like cells exist in castration-resistance prostate cells [10].

We performed in this study morphological and histological investigation of $\mathrm{CK} 18^{-/-}$prostate in both physiological and regenerative conditions. We reported here, against our original hypothesis, that CK18 may be required for the morphogenesis and regeneration of prostate; deletion of CK18 in a mouse model did not affect the normal morphology and histology of prostate. However, loss of CK18 indeed compromised the branching of regenerative DLP.

\section{Materials and Methods}

2.1. Experimental Animals. $\mathrm{CK} 18^{-/-}$mice were introduced from Jackson Laboratory originated deposited by Dr. M. Bishr Omary. Genotyping of $\mathrm{CK} 18^{-/-}$mice is performed as previously reported in [12]. All mice were housed in experimental animal center at Renji Hospital, Shanghai Jiaotong University, in pathogen-free environment with controlled temperature and humidity. Experimental procedures follow the guidelines and recommendations from Institutional Laboratory Animal Use and Care Committee (IACUC). For genotyping, $2 \mathrm{~mm}$ tail sample was obtained for genomic DNA extraction. $150 \mathrm{uL} 25 \mathrm{mM} \mathrm{NaOH} / 0.2 \mathrm{mM}$ EDTA was added to the tail sample and kept at $98^{\circ} \mathrm{C}$ for 1 hour, followed by neutralization with $150 \mathrm{uL}$ of $40 \mathrm{mM}$ Tris- $\mathrm{HCl}(\mathrm{pH}=$ 5.5). After a brief centrifugation, $2 \mu \mathrm{L}$ of supernatant was used for PCR reaction. The genotyping primers we used are $5^{\prime}$-AAGGAATCCAGGAAGGGAGA-3'; 5'-AGCCCCGGACTTACTTGACT- $3^{\prime}$, and $5^{\prime}$-GCCAGAGGCCACTTGTGTAG-3'. Thermal cycling parameters included (i) an denaturation at $95^{\circ} \mathrm{C}$ for $5 \mathrm{~min}$; (ii) 30 cycles of denaturation at $95^{\circ} \mathrm{C}$ for $30 \mathrm{~s}$, annealing at $60^{\circ} \mathrm{C}$ for $1 \mathrm{~min}$, and extension at $72^{\circ} \mathrm{C}$ for $1 \mathrm{~min}$; and (iii) a final extension at $72^{\circ} \mathrm{C}$ for $5 \mathrm{~min}$. The PCR products for mutant and wild-type allele are $187 \mathrm{bp}$ and $312 \mathrm{bp}$, respectively.

2.2. Hematoxylin and Eosin (HÆE) Staining. Prostate samples were fixed in $4 \%$ paraformaldehyde, dehydrated and paraffin embedded. After that, the paraffined prostates were cut at $5 \mu \mathrm{m}$ thickness on a microtome. Slides were stained with hematoxylin and eosin following standard protocols. Multiple fields were selected for each $\mathrm{H} \& \mathrm{E}$-stained section to compare the differences in prostate.

2.3. Immunoblotting. $100 \mu \mathrm{g}$ protein samples extracted from both wild-type and $\mathrm{CK} 18^{-/-}$knockout prostates were used for electrophoresis in 10\% sodium dodecyl sulfate polyacrylamide gel. Antibodies against CK18 (Abcam) were used as primary antibody.

2.4. Castration and Androgen Replacement. Four 2-monthold male mice of each genotype are used in our experiment. After anesthesia with avertin, the testes and epididymides were completely removed. The distal end of the spermatic cord was ligated with suture. 14 days after castration, a $0.8 \mathrm{~cm}$ sustained-release capsule of stanolone (30-40 mg, Sigma) was implanted subcutaneously. The mice were sacrificed for prostate collection 14 days later.

2.5. Prostate Microdissection. The separated prostate was moderately digested in $10 \mathrm{mg} / \mathrm{mL}$ collagenase for $10 \mathrm{~min}$. After digestion, microdissection was performed to visualize the individual ductal networks of prostate with fine forceps. All specimens were observed and photographed with microdissection microscope (Nikon SMZ800) for branch and tip quantification.

2.6. Immunofluorescence Staining. Immunofluorescence staining was performed with 8 um cryosections. After fixation in 4\% PFA at room temperature for $5 \mathrm{~min}$, sections were immerged in sodium citrate solution $(10 \mathrm{mM}, \mathrm{pH}=6.0)$ and microwaved for antigen retrieval for $5 \mathrm{~min}$. Sections were subsequently treated with $0.5 \%$ triton for $10 \mathrm{~min}$, followed by PBS wash and blocking with $10 \%$ normal goat serum (NGS) for 1 hour at room temperature. Specimens were then incubated with the primary antibodies overnight at $4^{\circ} \mathrm{C}$. Primary antibodies were washed at least three times with PBS containing 1\% NGS. After washing, secondary antibodies conjugated with Alexa-488 or 594 (Jackson Laboratory) were added to the specimens and incubated for 1 hour at room temperature. Secondary antibody was washed with PBS containing 1\% NGS 3 times. Slides were then mounted with DAPI containing mounting medium from Vector Laboratories. We used the followed primary antibodies in the immunofluorescence staining experiments: anti-p63 (Santa Cruz, sc-8431), anti-CK5 (Epitomics, 1988-1), anti-CK8 (Covance, MMS-162P), anti-CK8 (Epitomics, 2032-1), and anti-CK18 (Epitomics, 3258-1).

\section{Results}

3.1. H\&E Staining Shows No Changes in Histology of $\mathrm{CK} 18^{-/-}$ Mouse Prostates. To investigate the in vivo function of CK18 in prostate morphogenesis, we made use of $\mathrm{CK} 18^{-/-}$mice. Genomic PCR detected the null alleles in $\mathrm{CK}_{1}{ }^{-/-}$mice (Figure 1(a)). Immunoblotting further confirmed a complete CK18 deletion in prostates collected from knockout animals (Figure 2(a)). In order to assess the impact of CK18 deletion on the histology of developing prostate, we performed $\mathrm{H} \& \mathrm{E}$ staining of prostates from mutant and control mice at different postnatal development stages. Surprisingly we did not find any difference in gross appearance and histological structure between $\mathrm{CK} 18^{-/-}$and WT prostates. As shown in Figure 2, size of prostate from 8 -week-old $\mathrm{CK} 18^{-/-}$mice was comparable to WT. H\&E staining of mutant prostate AP, VP, and DLP did display a normal glandular structure and branching pattern (Figure 2). We examined carefully the prostate of 1-week-old and 4-week-old mutant mice as well, but no histological changes were detected (data not shown).

3.2. Both Luminal and Basal Cell Compartments Are Detected in the CK18 ${ }^{-/-}$Mouse Prostate. CK18 is a frequently used 


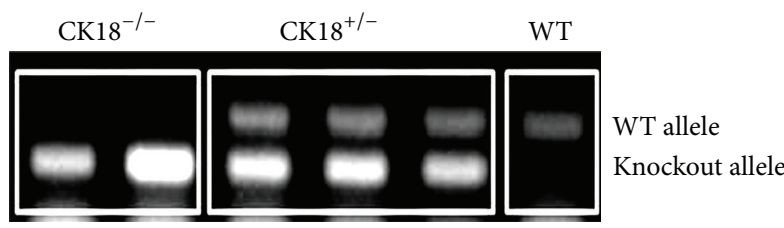

(a)

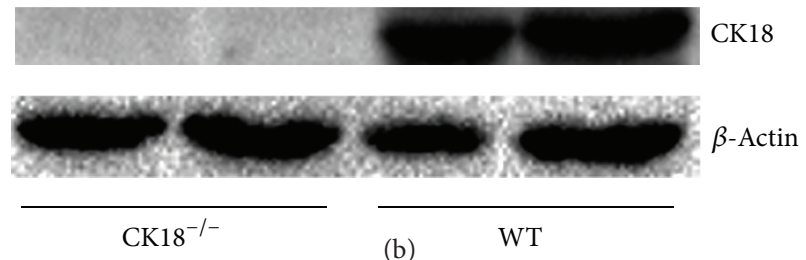

(b)

FIGURE 1: CK18 deletion in prostate from CK18 ${ }^{-/-}$mouse is verified by PCR and immunoblotting. (a) CK18 null allele and wild-type (WT) allele were detected by PCR analysis of tail samples. (b) Immunoblotting verifies ablation of CK18 expression in the CK18 ${ }^{-/-}$mouse prostate. Two mice of each genotype are used.

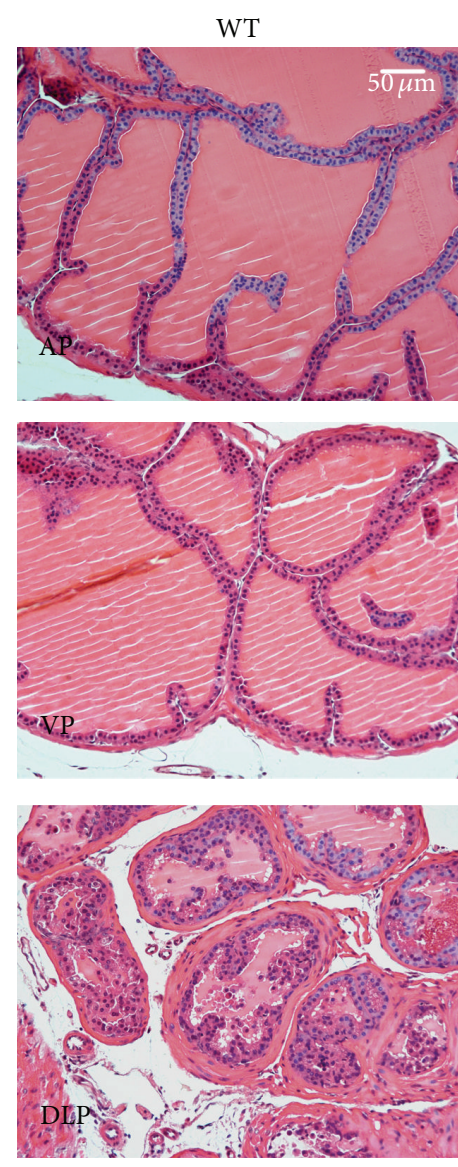

(a)
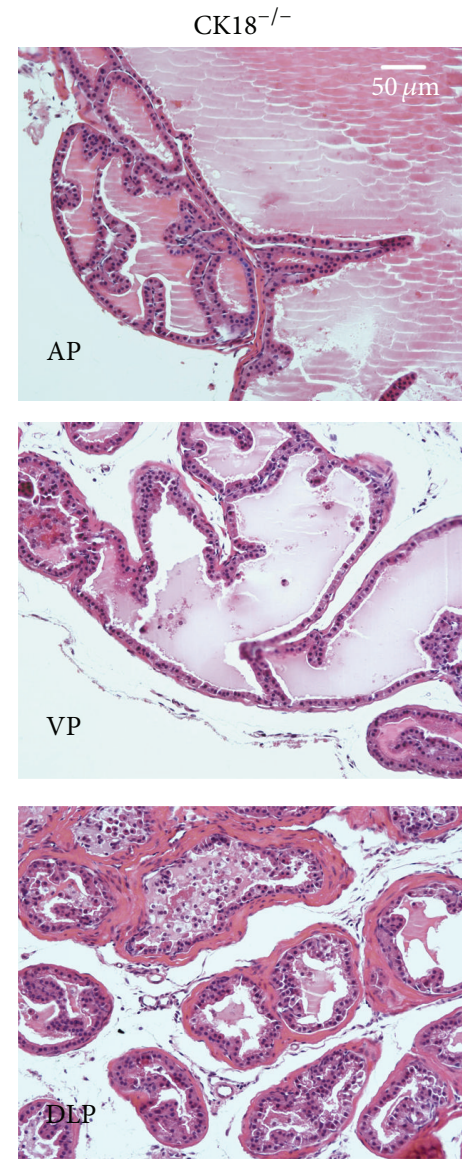

(b)

FIGURE 2: H\&E staining displays no histological difference between WT and CK18 ${ }^{-/-}$mouse prostates. Representative H\&E staining of AP, VP, and DLP prostates from WT (a) and $\mathrm{CK}_{1}{ }^{-/-}$(b) mice, shows normal histology of $\mathrm{CK} 18^{-/-}$prostates. Images were taken by Nikon light microscope (Nikon Eclipse Ti) with 200x magnification. Scale bars are $50 \mu \mathrm{m}$ for each related row.

luminal cell marker. To investigate if CK18 loss affects the luminal cell compartment, we performed immunofluorescence staining on mutant and WT prostates using wellcharacterized markers for luminal cells and basal cells. As expected, no expression of CK18 was detected from prostates of mutant mice (Figure 3). P63 and CK5 are selectively expressed in basal cells, while CK8, CK18, and AR are preferentially expressed in luminal cells. As shown in Figure 3, both luminal and basal cells were detected in $\mathrm{CK}_{1} 8^{-/-}$prostates, with comparable numbers to WT controls, implicating that CK18 is not required for luminal cell differentiation in the prostate.

3.3. CK18 Is Dispensable for Prostate Branching. Tubular morphogenesis of the mouse prostate completes at two months after birth. The maturation of AP, DP, and DLP accomplishes by then (Figures $4(\mathrm{a})$ and $4(\mathrm{~b})$ ). We further examined the function of CK18 in prostate tubular branching. The complex 
WT
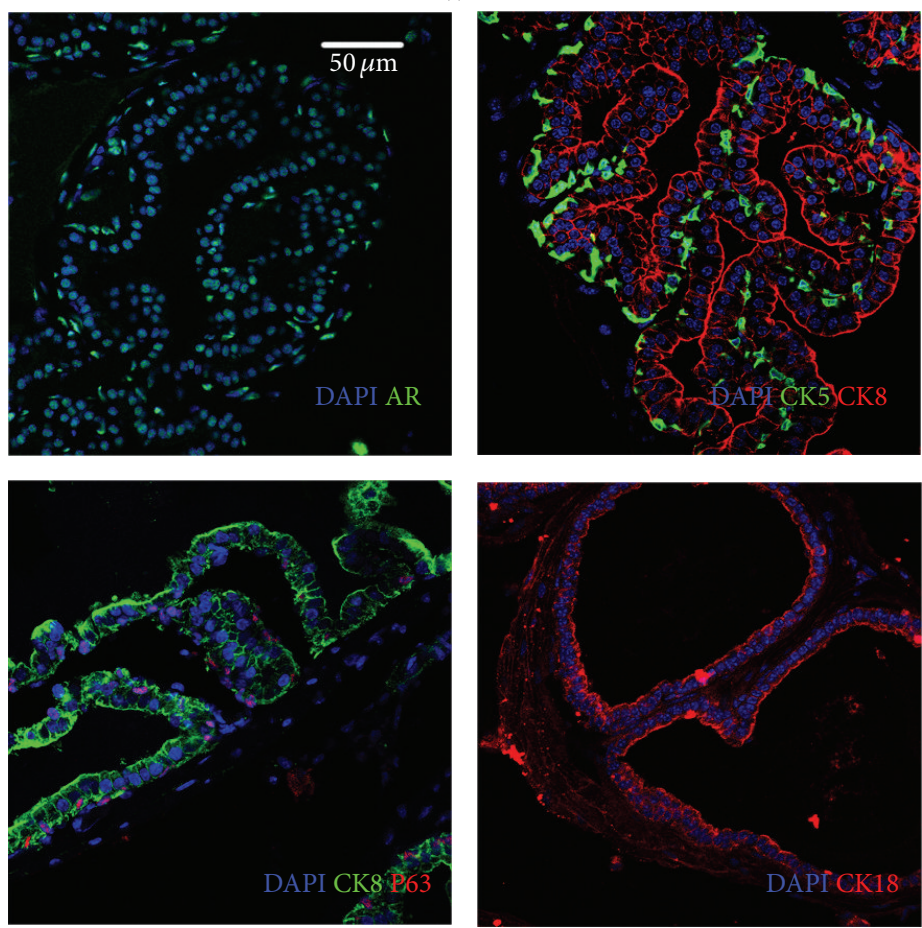

(a)
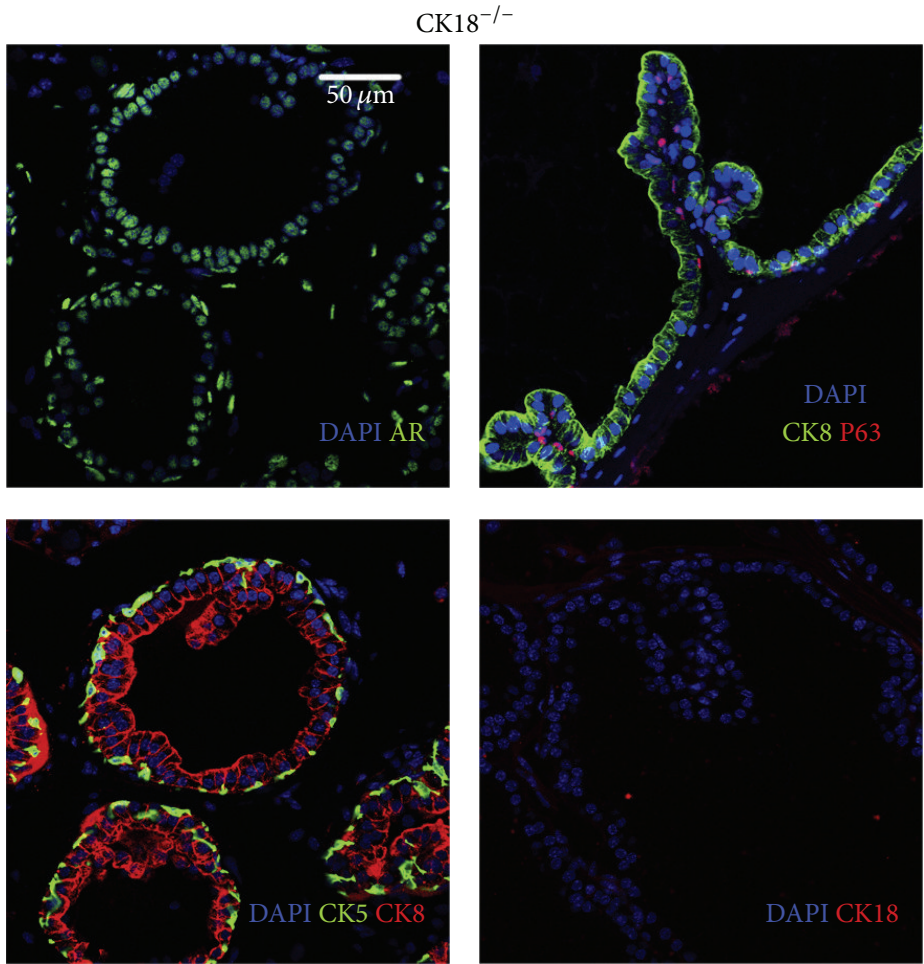

(b)

FIGURE 3: Normal basal and luminal cell compartments in $\mathrm{CK} 18^{-/-}$prostates. Immunofluorescence staining of prostate frozen sections displays similar luminal and basal cell compartments in WT (a) and CK18 ${ }^{-/-}$(b) prostates. Luminal cells are positive for AR, CK8, and CK18, while the basal cells are CK5 and P63 positive. At least 4 mice were sacrificed in each group for prostate collection, sectioning, and staining. Multiple fields were thoroughly examined under a microscope. Representative images are shown. Pictures were taken using a Nikon fluorescent microscope (Nikon Eclipse Ti) with 400x magnification. Scale bars are $50 \mu \mathrm{m}$ for each related row. 

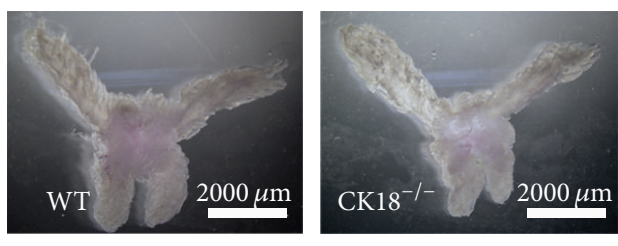

(a)

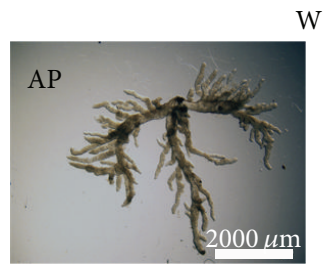

WT
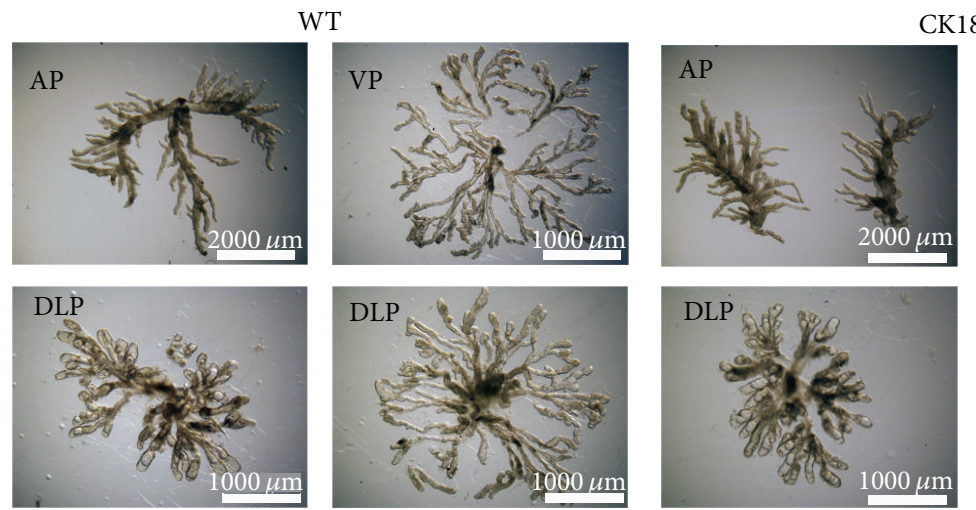

$\mathrm{CK} 18^{-1-}$

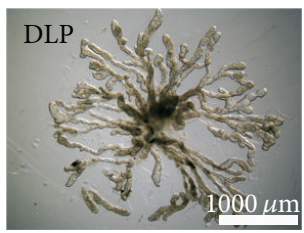

(b)
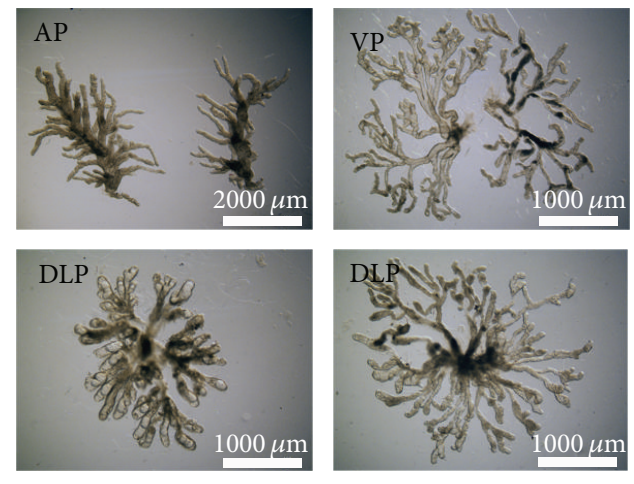

(c)

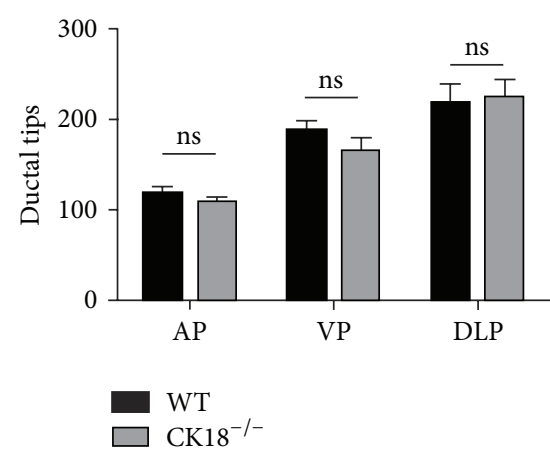

(d)

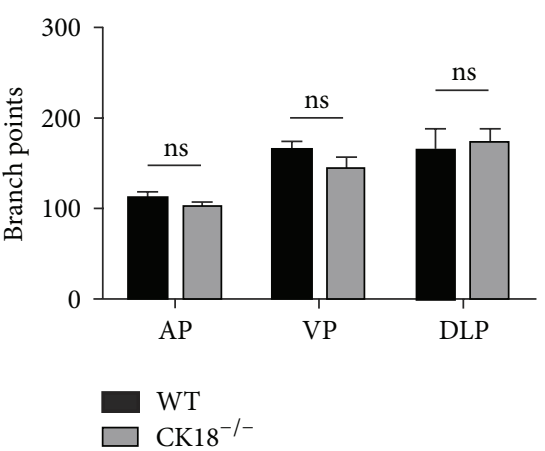

(e)

FIGURE 4: CK18 is dispensable for prostate branching. (a) The gross anatomic appearance of WT and CK18 ${ }^{-/-}$prostates with AP, VP, and DLP. Scale bars are $2000 \mu \mathrm{m}$. (b, c) The microdissection images display the ductal networks of prostate in left or right lobe of AP, VP, and DLP from WT and CK18 ${ }^{-1-}$ mice (a). Four mice of each group are used in the experiment. Only representative pictures are shown. Scale bar for AP image is $2000 \mu \mathrm{m}$, in VP and DLP images is $1000 \mu \mathrm{m}$. (d, e) Bar graph shows no significant differences of ductal tips and branch points in $\mathrm{AP}, \mathrm{VP}$, and DLP between WT and of CK18 ${ }^{-/-}$prostates $(n=4)$.

and extended prostatic ductal networks of all three lobes can be readily seen under microscope after brief digestion and careful microdissection (Figures 4(b) and 4(c)). The ductal tips and branching points were counted in each group to quantify the tubular formation. Statistical analysis showed no significant difference of ductal tips and branching points between mutant and WT groups, indicating normal tubular morphogenesis in $\mathrm{CK} 18^{-/-}$prostates.

3.4. CK18 Deletion Reduces the Branching of DLP during Prostate Regeneration Induced by Androgen Deprivation and Replacement. Androgen deprivation induces atrophy of prostate lobes, while administration of androgen efficiently stimulates prostate regeneration in rodents. To investigate if CK18 plays a role in a stress scenario such as prostate regeneration, we subjected both mutant and WT animals to castration and androgen replacement. Two-month-old mice from each group were used for castration. 14 days later, the castrated mice were given androgen by subcutaneous implants for another 14 days and then were sacrificed for prostate collection. Regenerated $\mathrm{CK}_{1} 8^{-/-}$prostates displayed similar gross anatomic appearances to control (Figure 5(a)). However, when we carefully microdissected each regenerated prostatic lobes, we did notice compromised tubular restoration in DLP from CK18 $8^{-/-}$mice (Figures 5(b) and $5(\mathrm{c}))$. Consistently, quantitative data showed significant reduction of both ductal tips and branch points in $\mathrm{CK} 18^{-/-}$ DLP compared to WT control, suggesting that CK18 does play a role in the prostate in response to stress and contributes to prostate regeneration. 

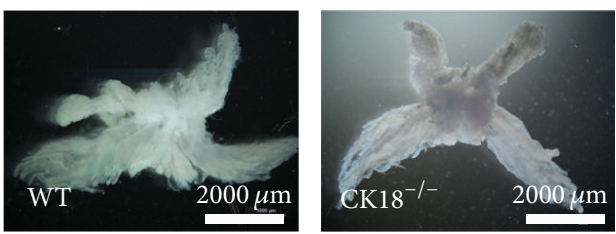

(a)

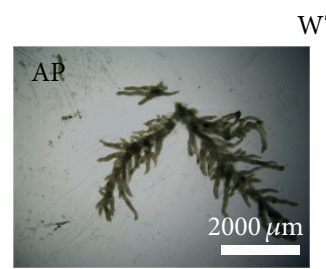

WT
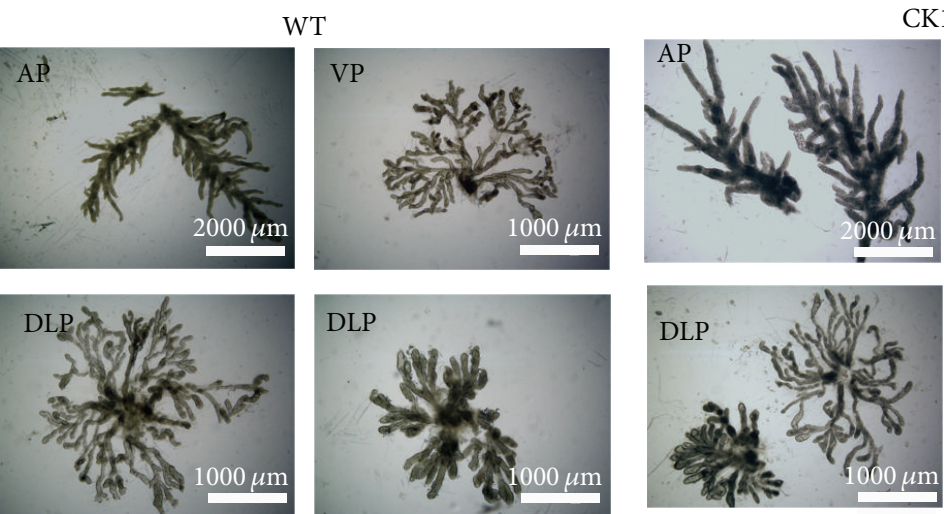

$\mathrm{CK} 18^{-/-}$
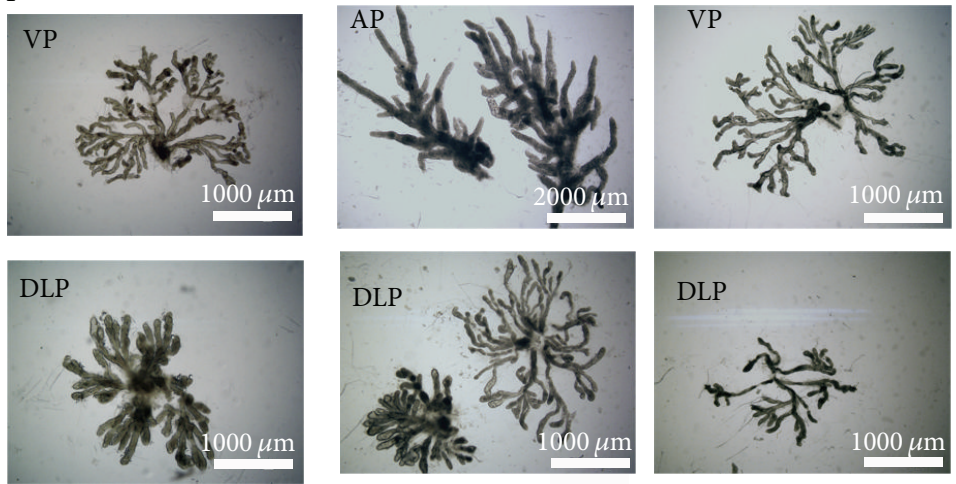

(b)

(c)

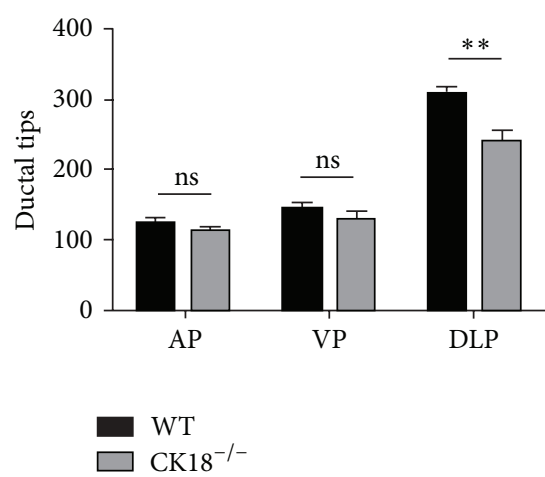

(d)

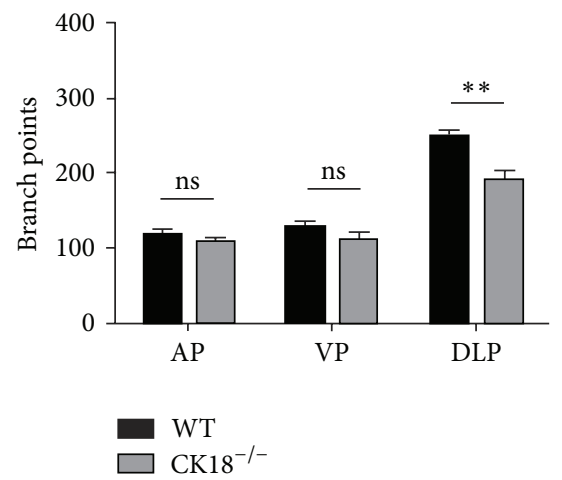

(e)

FIGURE 5: CK18 is required for DLP branching in prostate regeneration. (a) Prostates are carefully dissected after castration and androgen replacement. The gross anatomic appearance reveals no obvious difference between WT and CK18 ${ }^{-/-}$prostates. Scale bars are $2000 \mu \mathrm{m}$. $(\mathrm{b}$, c) The images of microdissected prostates from WT (a) and $\mathrm{CK}_{1}{ }^{-/-}$(b) mice after castration and androgen replacement show compromised branching pattern in DLP but not in VP and AP of $\mathrm{CK}_{1} 8^{-/-}$prostate. Four mice of each group are used in the experiment. Only representative pictures are shown. The scale bar in AP image is $2000 \mu \mathrm{m}$; scale bars in VP and DLP images are $1000 \mu \mathrm{m}$. (d, e) Bar graphs show significantly reduced umbers of ductal tips (mean $\pm \mathrm{SEM}$ ) and branch points in $\mathrm{CK} 18^{-/-}$DLP (mean $\pm \mathrm{SEM}$, ${ }^{* *}$ denotes $P<0.02$ ).

\section{Discussion}

The morphogenesis of developing prostate is governed by extracellular signal molecules and intracellular genetic programs [13-16]. For example, sonic hedgehog (Shh) pathway inhibits epithelial ductal branching of the prostate as reduced number of epithelial ducts was found in Shh-treated postnatal day 2 rat ventral prostate [17-19]. In contrast, castrated prostates could not regain normal size and structure after androgen replacement when the hedgehog pathway is blocked [20]. BMPs including BMP4 and BMP7 are additionally shown to be negative regulators in prostate ductal budding and branching [21, 22]. Basal-cell-specific transcriptional factor p63 is indispensable for prostate development.
UGS of $\mathrm{p} 63^{-/-}$mice is only able to generate prostatic structures with luminal and neuroendocrine cells but fails to give rise to any basal cells upon transplantation in renal capsule [4, 23]. CK18 is preferentially expressed in prostate luminal cells and has long served as luminal cell marker. We examined the morphology and histology of $\mathrm{CK} 18^{-/-}$prostates in this study to investigate the in vivo function of CK18 in prostate. To our surprise, CK18 deletion did not cause any morphological or histological change in homozygous ck18 knockout mice. However, when the prostate is under regenerative stress after castration and androgen replacement, CK18 ablation leads to partially compromised branching of DLPs.

CK18 and CK8 are the most abundant coexpressed keratins in mammals [24]. In the liver, CK18 and CK8 comprise 
the hepatocellular keratin, while CK18, CK8, and CK19 are the major keratins in hepatobiliary ductal cells [25]. Mutations of CK18 are associated with predisposition to subacute injury, apoptosis, and fibrosis of liver, possibly due to tempered CK18 phosphorylation and instability of filament structure [2527]. In epidermis, keratins are the major proteins constituting the cytoskeleton and protecting cell from mechanistic stress. CK5 and CK14 are often coexpressed as specific pairs in basal cell compartment. Deletion of CK14 in mice dramatically increases CK15 is that rarely expressed in basal cell [28]. Moreover, CK15 is able to reconstitute CK14 to form the keratin networks with CK5, implicating a redundant function of CK14 and CK15 in vivo. CK1 and CK10 are keratin pairs in suprabasal layer of epidermis. Similar phenomenon was also observed in $\mathrm{CK}_{10}^{-/-}$mice, in which upregulation of basal keratins 5 and 14 permits the normal epidermal differentiation of $\mathrm{CK}^{-/} 0^{-/}$mice [29]. Redundancy among CK6 $\alpha$ and CK6 $\beta 4$, CK4 and CK6, and CK19 and CK18 was further identified in the epidermis [30-32]. The result we show here that CK18 deletion barely affects prostate morphogenesis is probably also caused by upregulation of redundant cytokeratin. Increased CK19 expression is detected in $\mathrm{CK} 18^{-/-}$livers and double knockout of CK18 and CK19 led to much severe phenotypes including trophoblast fragility and severe early development defects [6, 32-34]. Therefore, we propose that CK19 which normally is not expressed in luminal cells may be upregulated in $\mathrm{CK} 18^{-/-}$prostates. However, embryonic lethality in the double knockout animals prevents us from the in vivo study of CK18 and CK19 redundancy. It is noteworthy that we did observe defects in $\mathrm{CK} 18^{-/-}$DLP branching morphogenesis when the prostate undergoes great stress during regeneration. Further analysis of expression level of other keratins in $\mathrm{CK} 18^{-/-}$prostate and experiment approaches like additional knocking down of relevant keratins will facilitate the identification of CK18 redundant protein in prostatic morphogenesis and regeneration.

\section{Conclusion}

In summary, our data suggest that although CK18 is an important marker for luminal cells, it has limited impact on the histology and ductal formation of the prostatic epithelium possibly due to functional redundancy of other keratins. However, in stress conditions such as regeneration process, deletion of CK18 partly compromises the branching and full recovery of DLP.

\section{Conflict of Interests}

The authors declare that there is no conflict of interests regarding the publication of this paper.

\section{Authors' Contribution}

Chenlu Zhang, Yanjing Guo, and Jian Cui contributed equally to the paper.

\section{Acknowledgments}

The study is supported by funds to W.-Q. Gao from the Chinese Ministry of Science and Technology (2012CB966800), the National Natural Science Foundation of China (NSFC) (81130038 and 81372189), Science and Technology Commission of Shanghai Municipality (Pujiang Program), Shanghai Health Bureau Key Disciplines and Specialties Foundation, Shanghai Education Committee Key Discipline and Specialties Foundation (J50208), KC Wong foundation, and Key Discipline and Specialty Foundation of Shanghai Municipal Commission of Health and Family Planning and funds to $\mathrm{H}$. $\mathrm{H}$. Zhu from the NSFC (81270627), Science and Technology Commission of Shanghai Municipality (Pujiang Program 12PJ1406100), and Shanghai Education Committee (12CG16, 13 YZ030 and young investigator program).

\section{References}

[1] A. Y. Nikitin, A. Matoso, and P. Roy-Burman, "Prostate stem cells and cancer," Histology and Histopathology, vol. 22, no. 9, pp. 1043-1049, 2007.

[2] A. Yang, R. Schweitzer, D. Sun et al., "p63 is essential for regenerative proliferation in limb, craniofacial and epithelial development," Nature, vol. 398, no. 6729, pp. 714-718, 1999.

[3] A. A. Mills, B. Zheng, X.-J. Wang, H. Vogel, D. R. Roop, and A. Bradley, "p63 is a p53 homologue required for limb and epidermal morphogenesis," Nature, vol. 398, no. 6729, pp. 708713, 1999.

[4] T. Kurita, R. T. Medina, A. A. Mills, and G. R. Cunha, "Role of p63 and basal cells in the prostate," Development, vol. 131, no. 20, pp. 4955-4964, 2004.

[5] Y. R. Weng, Y. Cui, and J. Y. Fang, "Biological functions of cytokeratin 18 in cancer," Molecular Cancer Research, vol. 10, no. 4, pp. 485-493, 2012.

[6] T. M. Magin, R. Schröder, S. Leitgeb et al., "Lessons from keratin 18 knockout mice: formation of novel keratin filaments, secondary loss of keratin 7 and accumulation of liver-specific keratin 8-positive aggregates," The Journal of Cell Biology, vol. 140, no. 6, pp. 1441-1451, 1998.

[7] P. C. Marker, A. A. Donjacour, R. Dahiya, and G. R. Cunha, "Hormonal, cellular, and molecular control of prostatic development," Developmental Biology, vol. 253, no. 2, pp. 165-174, 2003.

[8] Y. Sugimura, G. R. Cunha, and A. A. Donjacour, "Morphogenesis of ductal networks in the mouse prostate," Biology of Reproduction, vol. 34, no. 5, pp. 961-971, 1986.

[9] B. G. Timms, T. J. Mohs, and L. J. A. Didio, "Ductal budding and branching patterns in the developing prostate," The Journal of Urology, vol. 151, no. 5, pp. 1427-1432, 1994.

[10] A. Tsujimura, Y. Koikawa, S. Salm et al., "Proximal location of mouse prostate epithelial stem cells: a model of prostatic homeostasis," The Journal of Cell Biology, vol. 157, no. 7, pp. 12571265, 2002.

[11] Y. Sugimura, G. R. Cunha, and A. A. Donjacour, "Morphological and histological study of castration-induced degeneration and androgen-induced regeneration in the mouse prostate," Biology of Reproduction, vol. 34, no. 5, pp. 973-983, 1986.

[12] D. M. Toivola, M. I. Nieminen, M. Hesse et al., "Disturbances in hepatic cell-cycle regulation in mice with assembly-deficient keratins 8/18," Hepatology, vol. 34, no. 6, pp. 1174-1183, 2001. 
[13] J. J. Meeks and E. M. Schaeffer, "Genetic regulation of prostate development," Journal of Andrology, vol. 32, no. 3, pp. 210-217, 2011.

[14] G. R. Cunha, P. S. Cooke, and T. Kurita, "Role of stromalepithelial interactions in hormonal responses," Archives of Histology and Cytology, vol. 67, no. 5, pp. 417-434, 2004.

[15] G. S. Prins and O. Putz, "Molecular signaling pathways that regulate prostate gland development," Differentiation, vol. 76, no. 6, pp. 641-659, 2008.

[16] C. C. Pritchard and P. S. Nelson, "Gene expression profiling in the developing prostate," Differentiation, vol. 76, no. 6, pp. 624640, 2008.

[17] B.-E. Wang, J. Shou, S. Ross, H. Koeppen, F. J. de Sauvage, and W.-Q. Gao, "Inhibition of epithelial ductal branching in the prostate by sonic hedgehog is indirectly mediated by stromal cells," The Journal of Biological Chemistry, vol. 278, no. 20, pp. 18506-18513, 2003.

[18] S. H. Freestone, P. Marker, O. C. Grace et al., "Sonic hedgehog regulates prostatic growth and epithelial differentiation," Developmental Biology, vol. 264, no. 2, pp. 352-362, 2003.

[19] D. M. Berman, N. Desai, X. Wang et al., "Roles for Hedgehog signaling in androgen production and prostate ductal morphogenesis," Developmental Biology, vol. 267, no. 2, pp. 387-398, 2004.

[20] S. S. Karhadkar, G. S. Bova, N. Abdallah et al., "Hedgehog signalling in prostate regeneration, neoplasia and metastasis," Nature, vol. 431, no. 7009, pp. 707-712, 2004.

[21] M. L. G. Lamm, C. A. Podlasek, D. H. Barnett et al., "Mesenchymal factor bone morphogenetic protein 4 restricts ductal budding and branching morphogenesis in the developing prostate," Developmental Biology, vol. 232, no. 2, pp. 301-314, 2001.

[22] I. B. Grishina, S. Y. Kim, C. Ferrara, H. P. Makarenkova, and P. D. Walden, "BMP7 inhibits branching morphogenesis in the prostate gland and interferes with Notch signaling," Developmental Biology, vol. 288, no. 2, pp. 334-347, 2005.

[23] S. Signoretti, M. M. Pires, M. Lindauer et al., "p63 regulates commitment to the prostate cell lineage," Proceedings of the National Academy of Sciences of the United States of America, vol. 102, no. 32, pp. 11355-11360, 2005.

[24] N.-O. Ku, D. M. Toivola, Q. Zhou, G.-Z. Tao, B. Zhong, and M. B. Omary, "Studying simple epithelial keratins in cells and tissues," Methods in Cell Biology, vol. 78, pp. 489-517, 2004.

[25] N.-O. Ku, P. Strnad, B.-H. Zhong, G.-Z. Tao, and M. B. Omary, "Keratins let liver live: mutations predispose to liver disease and crosslinking generates Mallory-Denk bodies," Hepatology, vol. 46, no. 5, pp. 1639-1649, 2007.

[26] P. A. Coulombe and M. B. Omary, "Hard' and "soft" principles defining the structure, function and regulation of keratin intermediate filaments," Current Opinion in Cell Biology, vol. 14, no. 1, pp. 110-122, 2002.

[27] P. Strnad, Q. Zhou, S. Hanada et al., "Keratin variants predispose to acute liver failure and adverse outcome: race and ethnic associations," Gastroenterology, vol.139, no. 3, pp. 828.e3-835.e3, 2010.

[28] C. Lloyd, Q.-C. Yu, J. Cheng et al., “The basal keratin network of stratified squamous epithelia: defining K15 function in the absence of K14," The Journal of Cell Biology, vol. 129, no. 5, pp. 1329-1344, 1995.

[29] J. Reichelt, H. Bussow, C. Grund, and T. M. Magin, "Formation of a normal epidermis supported by increased stability of keratins 5 and 14 in keratin 10 null mice," Molecular Biology of the Cell, vol. 12, no. 6, pp. 1557-1568, 2001.
[30] S. M. Wojcik, M. A. Longley, and D. R. Roop, "Discovery of a novel murine keratin 6 (K6) isoform explains the absence of hair and nail defects in mice deficient for K6a and K6b," The Journal of Cell Biology, vol. 154, no. 3, pp. 619-630, 2001.

[31] S. L. Ness, W. Edelmann, T. D. Jenkins, W. Liedtke, A. K. Rustgi, and R. Kucherlapati, "Mouse keratin 4 is necessary for internal epithelial integrity," The Journal of Biological Chemistry, vol. 273, no. 37, pp. 23904-23911, 1998.

[32] M. Hesse, T. Franz, Y. Tamai, M. M. Taketo, and T. M. Magin, "Targeted deletion of keratins 18 and 19 leads to trophoblast fragility and early embryonic lethality," The EMBO Journal, vol. 19, no. 19, pp. 5060-5070, 2000.

[33] R. Moll, M. Divo, and L. Langbein, "The human keratins: biology and pathology," Histochemistry and Cell Biology, vol. 129, no. 6, pp. 705-733, 2008.

[34] T. M. Magin, P. Vijayaraj, and R. E. Leube, "Structural and regulatory functions of keratins," Experimental Cell Research, vol. 313, no. 10, pp. 2021-2032, 2007. 


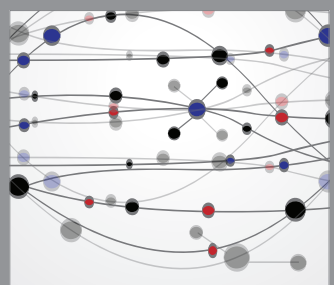

The Scientific World Journal
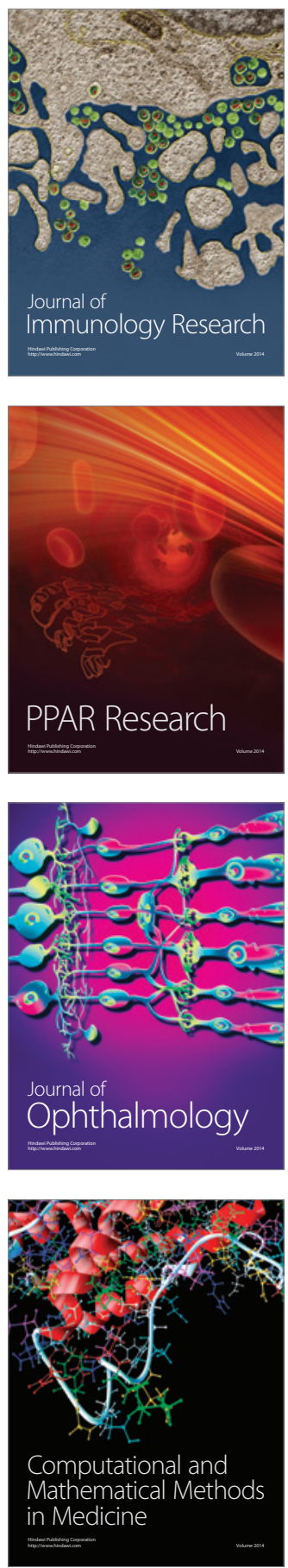

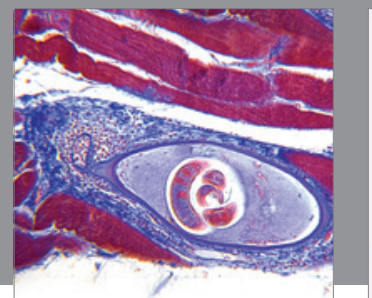

Gastroenterology

Research and Practice
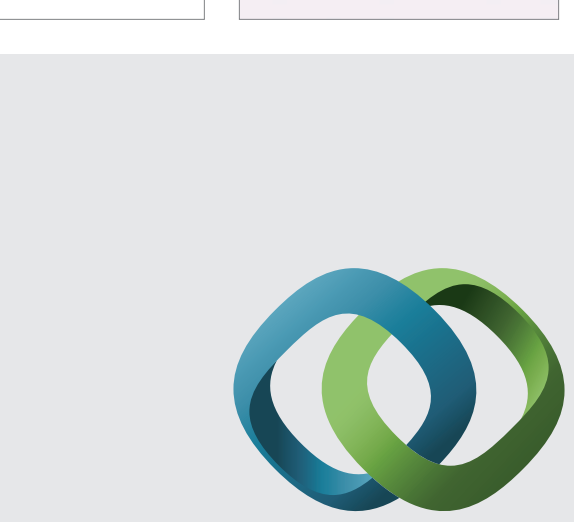

\section{Hindawi}

Submit your manuscripts at

http://www.hindawi.com
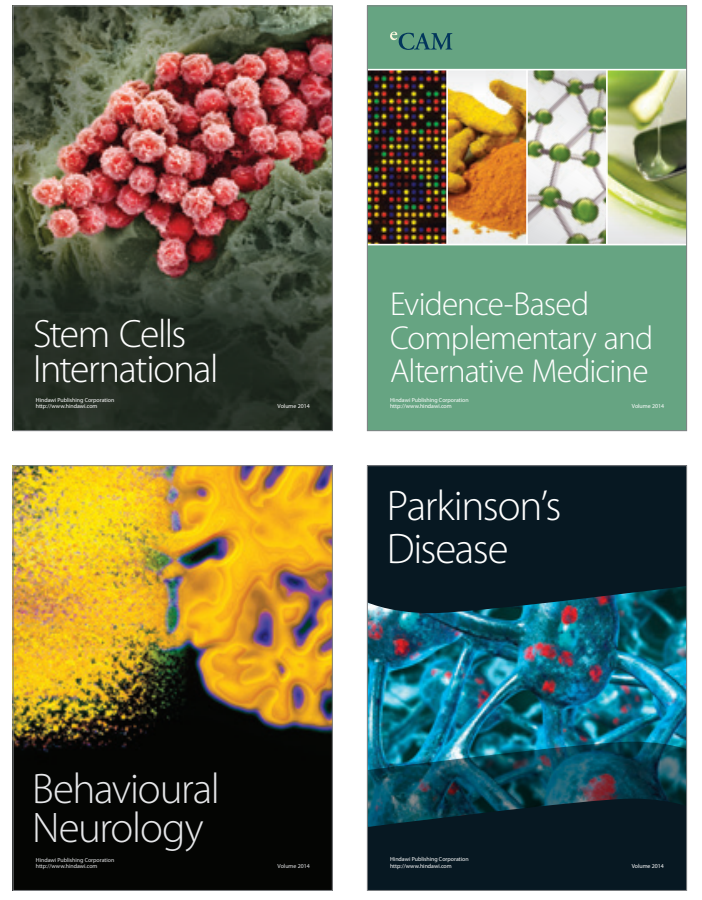
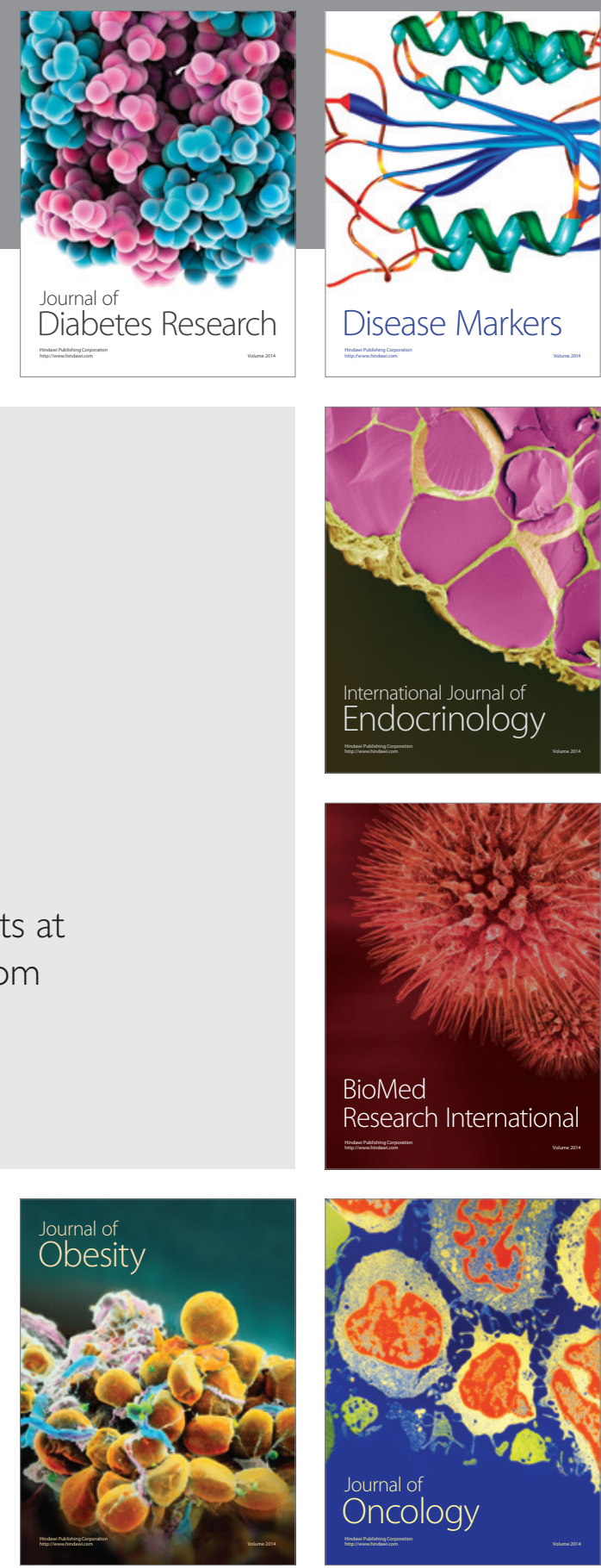

Disease Markers
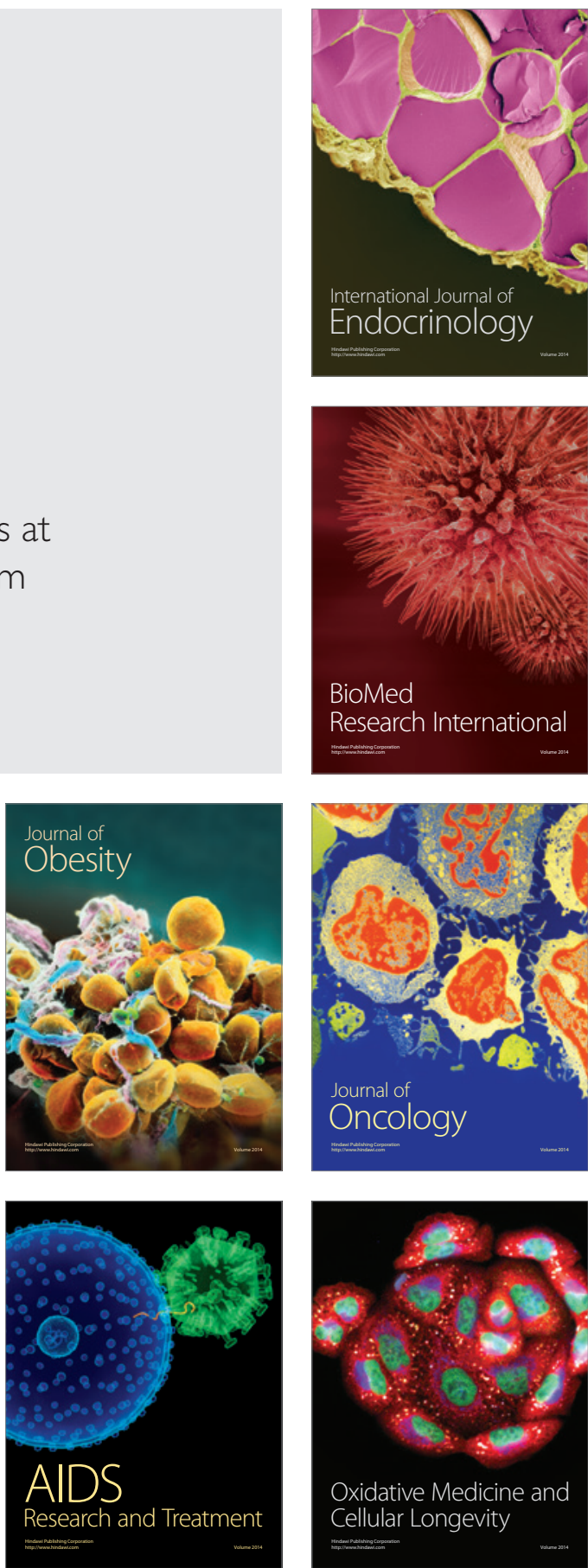